\title{
Planetary motion in double stars: the influence of the secondary
}

\author{
Elke Pilat-Lohinger \\ Institute for Astronomy, University of Vienna, Türkenschanzstrasse 17, A-1180 Vienna, \\ Austria \\ email: lohinger@astro.univie.ac.at
}

\begin{abstract}
Among more than 120 discovered exo-planets, less than 20 were found in double star systems. Out of this sample we studied the planetary motion in those systems that can be regarded as close binaries, i.e. HD41004 AB, $\gamma$ Cephei and Gliese 86. In this study we concentrate on the first two systems, where the secondaries are M4 V dwarfs at about 20 AU from the hoststar. A comparison of previous studies - where the dynamical behavior was studied in the (semimajor axis, inclination) plane (see Dvorak et al. 2003a) for $\gamma$ Cephei and Pilat-Lohinger \& Funk (2004) for HD41004 A) - shows significant differences in the stability maps. Our numerical investigation examines the region between 0.5 and $1.2 \mathrm{AU}$, which is influenced mainly by mean motion resonances when the initial position of the detected planet $a_{g p}<1.5 \mathrm{AU}$. If we move the planet farther away from the host-star (to distances $>1.5 \mathrm{AU}$ ) we observe an arc-shaped chaotic structure in the dynamical map.
\end{abstract}

Keywords. Binaries: close, celestial mechanics, methods: numerical

\section{Introduction}

Dynamical investigations of detected exo-planets in binaries are mainly restricted to the study of S-type motion (where the planet moves around one stellar component), whereas the P-type motion (where the planet revolves around both stars on a distant orbit) can not be applied yet, as no planet has been found in a very close binary. Moreover, there are only three binaries with distances of about $20 \mathrm{AU}$, all others have distances between 100 and 6000 AU (cf. Eggenberger et al. 2004). Previous studies of planetary motion in close binaries - i.e. $\gamma$ Cephei (cf. Dvorak et al. 2003a) and HD41004 AB (cf. Pilat-Lohinger \& Funk 2004) led to significantly different results although the systems seem to be similar, since in both cases the distance of the stellar components is about $20 \mathrm{AU}$ and the perturbing secondary is a M4 main sequence star of 0.4 solar-masses. The numerical stability study of fictitious planets in the examined (semi-major axis $\left(a_{f p}\right)$, inclination $(i)$ ) parameter space showed for $\gamma$ Cephei an influence of the secondary at low inclinations (see Section 3 and Dvorak et al. 2003a), which was not found for the system HD41004 AB, where this region is divided by mean motion resonances into several stable stripes (see Section 4 or Pilat-Lohinger \& Funk 2004).

In our numerical study we used for both exo-solar planetary systems the restricted four body problem (R4BP), that describes the motion of a massless planet in the gravitational field of three massive bodies, i.e. the binary and the detected giant planet. Furthermore, to see a potential effect of the secondary on the region between 0.5 and 1.2 $\mathrm{AU}$, we studied the same massless bodies in the restricted three body problem (R3BP) where we ignored the secondary. The orbital behavior was determined by means of the Fast Lyapunov Indicators (FLIs) (cf. Froeschlé et al. 1997). Moreover, in some cases we 
Table 1. Orbital parameters of the binary $\gamma$ Cephei (Cochran et al. 2002)

\begin{tabular}{lllc}
\hline & primary & secondary & planet \\
\hline mass [solar masses]: & 1.6 & 0.4 & .00168 \\
semi-major axis [AU]: & & 21.36 & 2.15 \\
eccentricity: & & 0.44 & 0.209 \\
period [years & & 70 & 2.47 ] \\
\hline
\end{tabular}

computed additionally the maximum eccentricity of the orbits. Based on the results of previous studies (cf. Dvorak et al. 2003a and Pilat-Lohinger \& Funk 2004) we examined how the variation of the initial semi-major axis of the giant planet $\left(a_{g p}\right)$ will influence the region between 0.5 and $1.2 \mathrm{AU}$ - this is shown in Section 5, where one can see the stability maps for different $a_{g p}$ in both systems.

\section{Numerical methods}

To define the dynamical state of the planetary orbits we used the Fast Lyapunov Indicator (cf. Froeschlé et al. 1997), which distinguishes qualitatively between regular and chaotic motion. This chaos indicator is defined as the norm of the largest tangent vector: $\psi(t)=\sup _{i}\left\|v_{i}(t)\right\|$ with $i=1, \ldots n$ ( $n$ denotes the dimension of the phase space), which grows exponentially for orbits in a chaotic region. The FLI program uses the Bulirsch-Stoer integration method; the integration time was $10^{4}$ periods of the binary.

Additionally, we carried out some straightforward orbital computations using the LIE integration method (see e.g. Lichtenegger 1984, or Hanslmeier \& Dvorak 1984) and determined the maximum eccentricity (MEC) of the fictitious planets over the whole integration time span, which was between $10^{5}$ and $10^{6}$ years. Such a supplementary study has already been conducted in previous investigations, where the so-called habitable zone $(\mathrm{HZ}) \dagger$ of several exo-solar planetary systems was analyzed (see e.g. Dvorak et al. 2003a and 2003b, Pilat-Lohinger \& Funk 2004, Erdi et al. 2004).

\section{Planetary motion in the binary $\gamma$ Cephei}

The double star system $\gamma$ Cephei can be found at a distance of about 11.8 pc from the Sun, where the detected Jupiter-like planet moves on an eccentric orbit around its hoststar (a K1 IV star). The computations have been carried out using the orbital parameters of Cochran et al., who discovered this planet in 2002 (see Table 1).

On the basis of a general stability study of S-type motion (cf. Pilat-Lohinger \& Dvorak 2002), which defines the border of the stable zone for $\gamma$ Cephei at about 3.6 AU, we were able to establish the stable motion for the discovered planet.

To determine regions where other fictitious planets might exist we used the R4BP as dynamical model, and found a stable zone between the host-star and the detected planet. The results of these computations are summarized in Fig. (1.a), where we varied the initial semi-major axis of the fictitious planet $\left(a_{f p}\right)$ from 0.5 to $1.8 \mathrm{AU}$ (x-axis) and increased its initial inclination from $0^{\circ}$ to $40^{\circ}$ on the y-axis. The dark region in Fig. (1.a) indicates chaotic motion and long-term stability is referred to the white region. Moreover, one can see a significant "chaotic path", which appears due to the fact that (i) we expect stronger perturbations of the secondary for low inclined orbits, and (ii) at about $1 \mathrm{AU}$

$\dagger$ The habitable zone of a Sun-like star is, roughly speaking, located at a distance from the star where liquid water can exist on the surface of a terrestrial-like planet. 

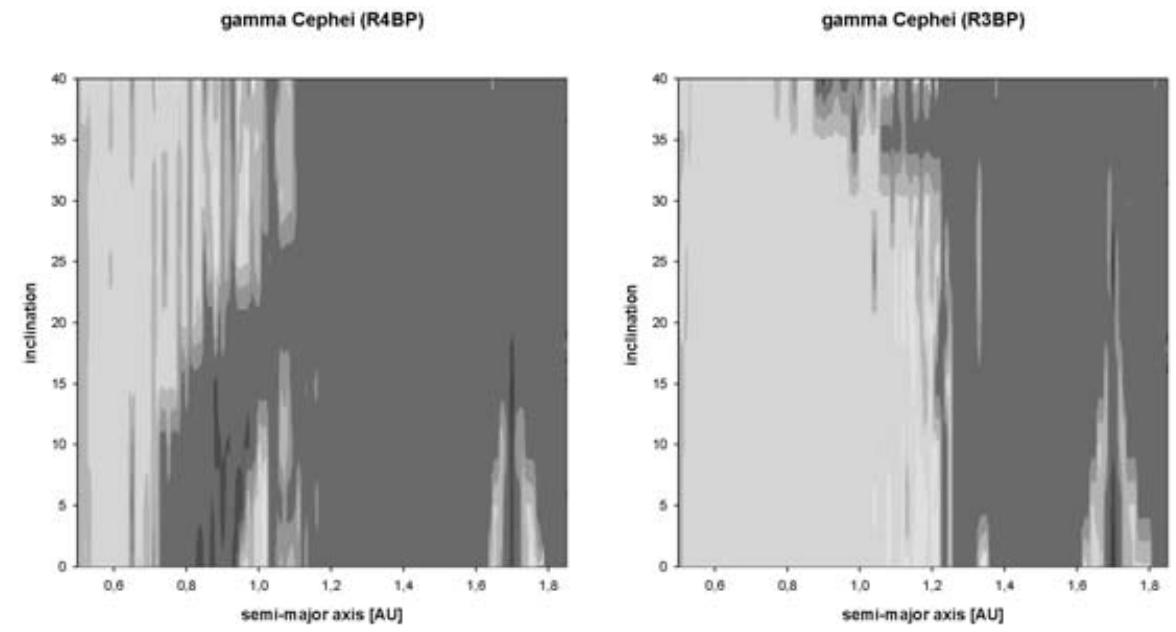

Figure 1. Stability maps for a fictitious planet in the vicinity of $\gamma$ Cephei: left panel (a) shows the result in the R4BP (i.e. $\gamma$ Cephei + secondary + detected planet + fictitious planet) and right panel (b) shows the result in the R3BP (i.e. $\gamma$ Cephei + detected planet + fictitious planet). The dark region shows the chaotic zone and the white area the stable one; for details see the text.

a small stable island remains, which is close to the $3: 1$ mean motion resonance. This stable island appears for all chosen mean anomalies $\left(0^{\circ}, 45^{\circ}, 90^{\circ}, 135^{\circ}, 180^{\circ}, 225^{\circ}, 270^{\circ}\right.$ and $315^{\circ}$ ) of the fictitious planets, so that we suspect a stabilization of this region due to the resonance. Fig. (1.b) shows the same dynamical map for the computations in the R3BP (where we neglected the secondary). In this model one can see a much larger stable region with a well defined border. Only for high inclinations $\left(>30^{\circ}\right)$ a reduction of the stable zone occurs due to the Kozai resonance. A comparison of the two dynamical maps led to the assumption that the arc-shaped chaotic structure could result from secular perturbations, but this has still to be proved. The two stable islands between 1.6 and 1.8 AU seem to be influenced also by the secondary, since they are smaller in size in the R4BP (Fig. 1.a).

\section{Planetary motion in HD41004 AB}

The study of planetary motion in the close binary HD41004 AB was carried out to examine the dynamical state of terrestrial-like planets in the habitable zone (HZ) of HD41004 A $\dagger$, which is according to Kasting et al. (1993) between 0.48 and nearly 1 AU. A planetary companion for HD41004 A was announced by M. Mayor in July 2003 during the XIXth IAP Colloquium on "Extra-solar planets today and tomorrow" in Paris, but the system parameters (see Table 2) show that the study of this system is actually more complicated, since the secondary is accompanied by a brown dwarf of $19 \mathrm{~m}_{\text {Jup }}$. However, similar dynamical studies for this exo-solar planetary system like the ones for $\gamma$ Cephei in the R4BP and R3BP allowed a simplification of the investigation of the HZ of HD41004 A, since the two dynamical maps (Figs. (2.a) and (2.b)) do not indicate a significant difference in the (a,i)-plane, which probably means that this region is not under a strong influence of the secondary. In both figures one can see the same structure of the HZ, which is divided into stable stripes by mean motion resonances (1:4 near 0.52 AU, 7:2 near 0.57 AU, 3:1 around 0.63 AU and 8:3 near 0.68 AU), like the asteroid

$\dagger$ A detailed study of the HZ of HD41004 A will be presented in Pilat-Lohinger \& Funk 2004) 
HDA1004A (R4BP)

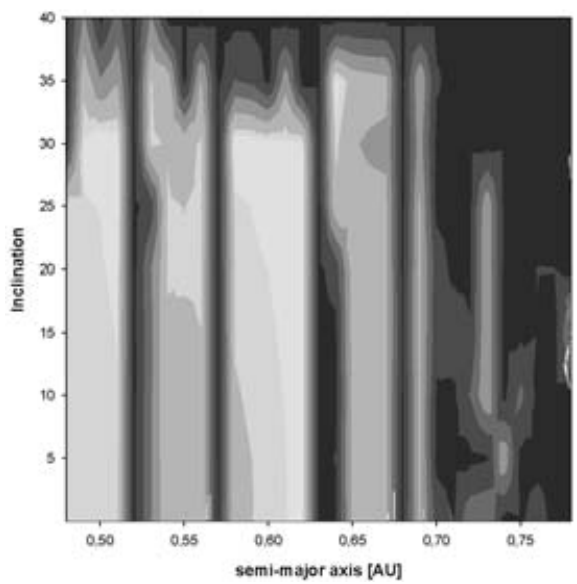

HD41004A (R3BP)

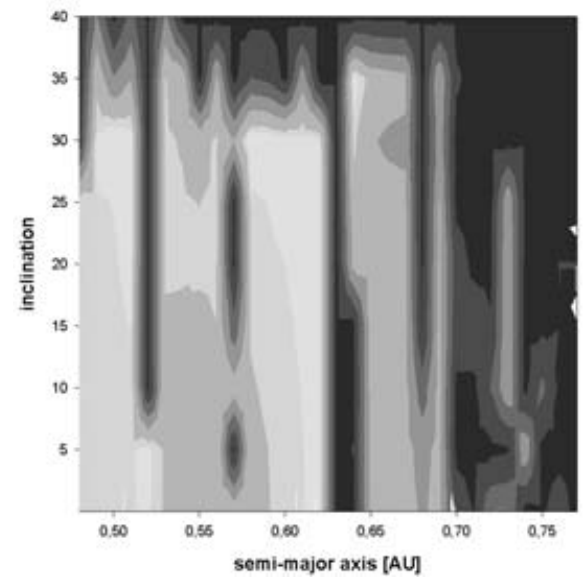

Figure 2. Stability maps for a fictitious planet in the HZ of HD41004 A: left panel (a) shows the result in the R4BP (i.e. binary HD41004 AB + detected planet + fictitious planet) and right panel (b) shows the result in the R3BP (i.e. HD41004 A + detected planet + fictitious planet). The different gray shades indicate the zones of different eccentricities: $e<0.2$ (white), $0.2<e<0.3$ (light gray), $\ldots, e>0.8$ (black, i.e. unstable region). Note that only the part of the HZ, where stable motion occurs, is plotted.

Table 2. Orbital parameters of the binary HD41004 AB (Zucker et al. 2004)

\begin{tabular}{llllc}
\hline & primary & secondary & brown dwarf & planet \\
\hline mass [solar masses]: & 0.7 & 0.4 & 0.01814 & 0.002196 \\
semi-major axis [AU]: & & $\sim 22$ & $\sim 22$ & 1.31 \\
eccentricity: & & $?$ & 0 & $0.39 \pm 0.17$ \\
period [years] & & $\sim 70$ & $\sim 70$ & $\sim 1.8$ \\
\hline
\end{tabular}

main-belt in our solar system. The left panel shows the stable motion (white region) in the R4BP, where the binary's eccentricity was 0.2 , and the right panel summarizes the motion in the R3BP, where the secondary was neglected. In both cases we determined the dynamical behavior by means of the FLIs and studied additionally the maximum eccentricity (MEC), to cancel out high eccentric motion $(e>0.5)$, in order to be sure that most of the orbits will be in th HZ. Even if the simplified system of HD41004 AB (i.e. without brown dwarf) seems to be quite similar as $\gamma$ Cephei, we found the contrary from our results (compare Figs. (1.a and b) and (2.a and b)). The orbital parameters of Tables 1 and 2 show that the most significant difference of the two exo-planetary systems is the position of the detected giant planet. Therefore, we studied both systems in the model of the R4BP, where we varied the initial $a_{g p}$, in order to find an explanation for this difference in the results.

\section{Stability study for different $a_{g p}$}

We computed the motion of fictitious bodies in the region between 0.5 and $1 \mathrm{AU}$ for the following positions of the giant planet: 1.3 AU, 1.5 AU, 1.7 AU, 1.9 AU and 2.1 AU. The most important results of the FLI computations are summarized in Figs. (3.a and $b$ ) and (4.a and b), where the white region denotes stable motion and the dark 

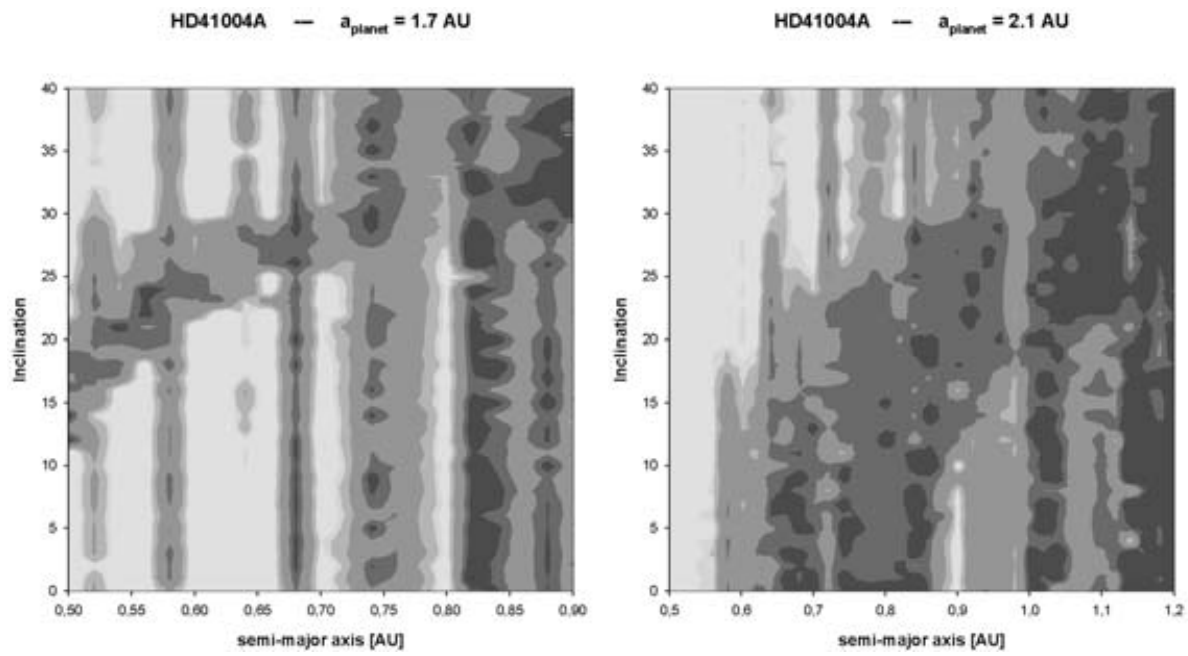

Figure 3. Stability maps for the HZ of HD41004 A with the detected giant planet moving at different distances from its host-star: (a) $a_{g p}=1.7 \mathrm{AU}$ and (b) $a_{g p}=2.1 \mathrm{AU}$. The dynamical behavior was determined by means of the FLIs, where white zones denote stable motion and dark region corresponds to chaotic motion (for more details see Section 5).
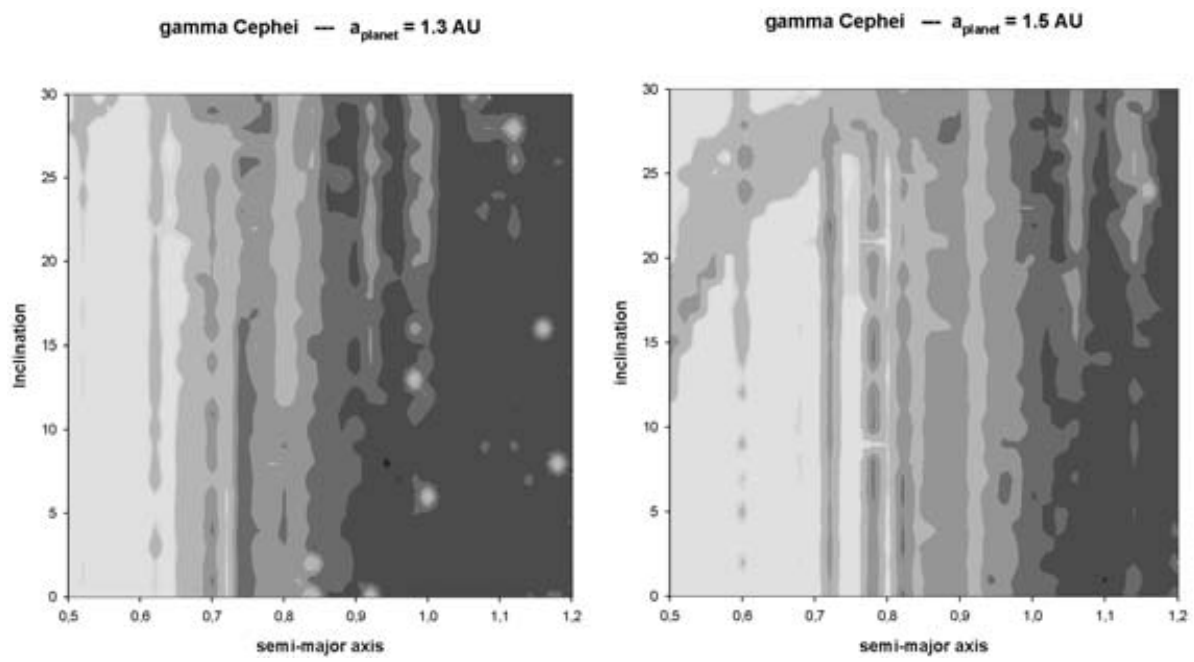

Figure 4. Stability maps for the $\mathrm{HZ}$ of $\gamma$ Cephei, with the detected giant planet moving at different distances from its host-star: (a) $a_{g p}=1.3 \mathrm{AU}$ and (b) $a_{g p}=1.5 \mathrm{AU}$. The dynamical behavior was determined by means of the FLIs, where white zones denote stable motion and dark region corresponds to chaotic motion (for more details see Section 5).

region indicates chaotic motion. One can see, that for $a_{g p}=1.3 \mathrm{AU}$ (see Figs. (2.a) for $\mathrm{HD} 41004 \mathrm{~A}$ and (4.a) for $\gamma$ Cephei) the region in the (semi-major axis, inclination)-map is influenced mainly by mean motion resonances (dark straight lines), while the chaotic arc-like structure appears only in the dynamical maps, where $a_{g p} \geqslant 1.5 \mathrm{AU}$. Since we expect stronger perturbations from the secondary on the giant planet, if it is moved toward the secondary, we assume that this curved chaotic shape results from secular perturbations (which has still to be proved). Following the sequence of Figs. (4.b), (3.a) and (3.b) one can see how the chaotic arc changes if $a_{g p}$ is closer and closer to the value corresponding to the secondary. 


\section{Conclusions}

Summarizing the numerical study of planetary motion in the region between 0.5 and 1 AU from a host-star, where we have to expect planetary and stellar perturbations, we can conclude the following:

(a) when the giant planet is close enough to the host-star (i.e. $<1.5 \mathrm{AU}$ for the two binary systems) the region seems to be only perturbed by the giant planet of the system and the stable zone is divided into several stable striped due to to mean motion resonances;

(b) when the position of the giant planet is $\geqslant 1.5 \mathrm{AU}$ from the host-star we assume perturbations of the secondary on the planet, which causes an arc-shaped chaotic structure probably due to secular perturbations. Since all massive bodies move in the same plane, we expect a precession of the perihelion of the giant planet.

Even if we use the same position of the giant planet in the two binary systems, we cannot find exactly the same dynamical map:

(i) for $a_{g p}=1.3 \mathrm{AU}$ one can see that the splitting of the stable zone into several stripes due to mean motion resonances with respect to the giant planet is much more clearly visible for HD41004 AB;

(ii) the arc-like chaotic structure appears earlier with the binary $\gamma$ Cephei (when $a_{g p}=1.5 \mathrm{AU}$ ), than for HD41004 A (when $a_{g p}=1.7 \mathrm{AU}$ );

(iii) for $a_{g p}=2.1 \mathrm{AU}$ the arched chaotic structure (also called chaotic path in Dvorak et al. 2003a) is more distinct in the binary $\gamma$ Cephei.

Therefore, we conclude that we have to study also other parameters like:

- the eccentricity of the binary;

- the mass-ratio of the binary;

- the mass of the giant planet.

which might change the dynamical maps as well.

A more detailed study is in progress and will be presented elsewhere.

\section{Acknowledgements}

The author wishes to acknowledge the support by the Austrian FWF (Hertha Firnberg Project T122) and wants to thank Dr. Z. Knezevic for his critical reading of the manuscript.

\section{References}

Cochran, W.D., Hatzes, A.P., Endl, M., Paulson, D.B., Walker, G.A.H, Campbell, B. and Yang, S. 2002, Astron. Astrophys. Suppl. Ser. 34, 916

Dvorak, R., Pilat-Lohinger, E., Funk, B. and Freistetter, F. 2003a Astron. Astrophys. 398, L1

Dvorak, R., Pilat-Lohinger, E., Funk, B. and Freistetter, F. 2003b, Astron. Astrophys. 410, L13

Eggenberger, A., Udry, S. and Mayor, M. 2004, Astron. Astrophys. 417, 353

Erdi, B., Dvorak, R., Sandor, Z., Pilat-Lohinger, E. and Funk, B. 2004, Mon. Not. R. Astron. Soc. 351, 1043

Froeschlé, C., Lega, E. and Gonczi, R. 1997, Cel. Mech. Dyn. Astron. 67, 41

Hanslmeier, A. and Dvorak, R. 1984, Astron. Astrophys. 132, 203

Lichtenegger, H. 1984, Celest. Mech. 34, 357

Pilat-Lohinger, E. and Dvorak, R. 2002, Cel. Mech. Dyn. Astron. 82, 143

Pilat-Lohinger, E. and Funk, B. 2004, "A stability study of the habitable zone of HD41004 A", in preparation

Zucker, S., Mazeh, T., Santos, N., Udry, S. and Mayor, M. 2004, Astron. Astrophys. 426, 695 